\title{
Geometry of Equisingular Families of Curves
}

\author{
Gert-Martin Greuel and Eugenii Shustin * \\ Dedicated to C.T.C. Wall on the occasion of his 60th birthday
}

\section{Contents}

$\begin{array}{ll}\text { Introduction } & 1\end{array}$

1 Existence of plane curves with given singularities $\quad 2$

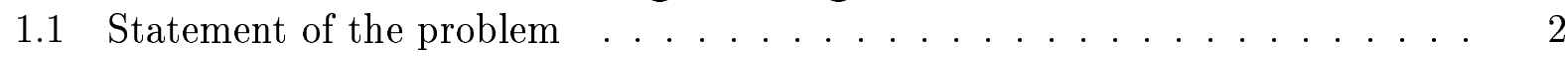

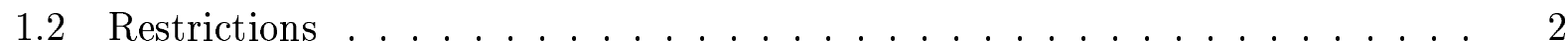

1.3 Some previously known constructions . . . . . . . . . . . . . . 3

1.4 Asymptotically optimal sufficient existence conditions . . . . . . . . . . . . 4

1.5 Zero-dimensional schemes associated with topological singularities . . . . . . 5

$1.6 h^{1}$-vanishing criteria for zero-dimensional schemes in the plane . . . . . . 6

1.7 Gluing singular points . . . . . . . . . . . . . . 7

2 Smoothness of equisingular and equianalytic families $\quad 8$

$2.1 \quad T$-property . . . . . . . . . . . . . . . . . 8

2.2 Curves on general surfaces . . . . . . . . . . . . . . 10

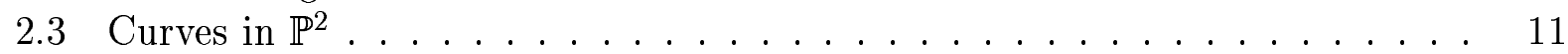

2.4 Curves on special surfaces . . . . . . . . . . . . . . 12

3 Irreducibility of equisingular families $\quad 13$

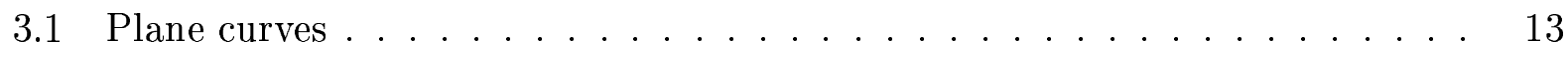

3.2 Curves on rational surfaces . . . . . . . . . . . . . 16

4 Perspectives and problems $\quad 16$

$\begin{array}{ll}\text { References } & 21\end{array}$

*The authors were supported by Grant No. G 039-304.01/95 from the German Israeli Foundation for Scientific Research and Development and by the DFG-Schwerpunkt "Algorithmische Zahlentheorie und Algebra" 


\section{Introduction}

Singular algebraic curves, their existence, deformation, families (from the local and global point of view) attract continuous attention of algebraic geometers since the last century. The aim of our paper is to give an account of results, new trends and bibliography related to the geometry of equisingular families of algebraic curves on smooth algebraic surfaces over an algebraically closed field of characteristic zero. This theory is founded in basic works of Plücker, Severi, Segre, Zariski, and has tight links and finds important applications in singularity theory, topology of complex algebraic curves and surfaces, and in real algebraic geometry.

We concentrate around the following traditionally stated questions. Given some classification of isolated curve singularities we call an equivalence class a type. Then the discriminant in the linear system $|D|$ on a smooth algebraic surface $\Sigma$ is decomposed into the set of non-reduced or reducible curves and the sets $V\left(D ; S_{1}, \ldots, S_{r}\right)$ of (irreducible) curves in $|D|$ having $r$ singular points of types $S_{1}, \ldots, S_{r}$ as their only singular points (in the sequel these sets are referred to as equisingular families - ESF). We ask:

- Is $V\left(D ; S_{1}, \ldots, S_{r}\right)$ non-empty?

- Is $V\left(D ; S_{1}, \ldots, S_{r}\right)$ smooth and has a "regular", or "expected", codimension in $|D|$ (expressed in terms of $\left.S_{1}, \ldots, S_{r}\right)$ ?

- What is the adjacency relation of ESF in the given $|D|$ (i.e. what are the deformations of a curve $C \in|D|$ ) ? Is this adjacency regular (i.e. do deformations of $C$ depend only on the local structure of its singular points)?

- Is $V\left(D ; S_{1}, \ldots, S_{r}\right)$ irreducible?

Among the variety of results related to the problems stated, we discuss basically sufficient conditions of numerical kind (i.e. expressed via numerical invariants of surfaces, curves, singularities), which ensure affirmative answers to the questions listed above for the given ESF. The case of plane curves is central in the theory and takes the main part in our paper. In fact, results on curves on other surfaces appeared as extensions and generalizations of the corresponding ones for plane curves.

As one of the most important ranges of applications we mention various constructions based on deformation theory. Especially this concerns Hilbert's 16th problem, the classification of real algebraic curves. First of all, the real version of Severi's theorem on the independence of deformations of nodes of plane curves, given by Brusotti [12], was a basis of classical constructions of real non-singular algebraic curves by Harnack, Hilbert, Brusotti, Wiman [37]. Using generalizations of this method on certain surfaces, Gudkov [37] classified real algebraic curves of small degree on quadrics, and recently Pecker [54] proved that any $g$ between zero and the Eisenbud-Harris upper bound $\pi_{d}(m, \alpha)$ is the genus of a non-singular curve in $\mathbb{P}^{m}$ of degree $d$, not lying on a hypersurface of small degree. The Viro method [84], [85], [86], being invented for the construction of real non-singular algebraic curves, brought new ideas into the deformation theory. Combining it with the independence of singular point deformations and criteria for the smoothness of ESF, one can

- construct curves (real or complex) with prescribed collection of singularities [67], [29], [72] (see section 2 below for more details) and prescribed topology [57] [62]; 
- classify deformations of certain singular points [63], [65], [64];

- construct interesting topological objects (see, for instance, in [21] counterexamples to one of Kirby's conjecture).

The material is organized in four sections: 1 . Existence of curves with given singularities (or, equivalently, non-emptiness of ESF), 2. Smoothness of ESF and independence of singular point deformations, 3. Irreducibility of ESF, 4. Open questions.

The authors would like to thank C. Lossen for his help during the preparation of this article.

\section{Existence of plane curves with given singularities}

\subsection{Statement of the problem}

The question, what are possible singularities of a complex plane algebraic curve of a given degree, is classical and can be traced back to Euler, Plücker, Segre, Severi. We consider this problem in two aspects: analytic and topological.

Let $(C, z),\left(C^{\prime}, z^{\prime}\right)$ be germs of complex plane algebroid curves with isolated singular points $z, z^{\prime}$. They are called analytically (topologically) equivalent if there is a local analytic diffeomorphism (homeomorphism) of neighborhoods of $z, z^{\prime}$ in the plane taking $(C, z)$ into $\left(C^{\prime}, z^{\prime}\right)$. The analytic equivalence can be expressed as an isomorphism of the complete local rings

$$
\hat{\mathcal{O}}_{C, z} \cong \hat{\mathcal{O}}_{C^{\prime}, z^{\prime}} \text {. }
$$

The topological equivalence is completely characterized by the following discrete invariants (see [92], [88], [77], [9]): the embedded resolution tree of $(C, z)$, the multiplicities of the strict transforms of $(C, z)$ at infinitely near points (including $z$ ) and the intersection numbers of the strict transforms of $C$ with the corresponding exceptional divisors.

Analytic or topological equivalence classes of isolated singular points are called analytic or topological singularities. So, the general problem is: given an integer $d>0$ and singularities (analytic or topological) $S_{1}, \ldots, S_{r}$, does there exist an irreducible curve of degree $d$ having $r$ singular points of types $S_{1}, \ldots, S_{r}$, respectively, as its only singularities ?

An important particular case is the same problem for one singularity. Namely, let $S$ be an analytic or topological singularity. What is the minimal degree $d(S)$ of a curve having a singular point of type $S$ ? In other words, we ask about a normal form of minimal degree of the given singularity.

\subsection{Restrictions}

Severi's theorem [61] that the inequality

$$
n \leq \frac{(d-1)(d-2)}{2}
$$

is necessary and sufficient for the existence of an irreducible curve of degree $d$ with exactly $n$ nodes, is the only complete answer in this problem. For other singularities, starting with ordinary cusps, the problem is still open except the case of degrees $\leq 6$, see [36], [89], [90], [16], [17], [79], [80], [81]. 
There are known various restrictions of type

$$
\sum_{i=1}^{r} \alpha\left(S_{i}\right) \leq a d^{2}+o\left(d^{2}\right)
$$

as necessary conditions for the existence of a curve of degree $d$ with $r$ singular points of types (topological or analytic) $S_{1}, \ldots, S_{r}$, where $\alpha$ is some positive invariant of the singularities, $a=$ const $>0$ (see a number of such inequalities both for arbitrary singularities, or for certain classes of them in [10], [42], [44], [56], [82]). We mention here the classical ones:

$$
\begin{gathered}
\sum_{i=1}^{r} \delta\left(S_{i}\right) \leq \frac{(d-1)(d-2)}{2}, \\
\sum_{i=1}^{r} \mu\left(S_{i}\right) \leq(d-1)^{2},
\end{gathered}
$$

where $\delta$ is the $\delta$-invariant and $\mu$ is the Milnor number.

In the case of one singularity there is a lower bound on the degree $d(S)$ of a normal form of the singularity $S$, coming from (1.2):

$$
d(S) \geq \sqrt{\mu(S)}+1
$$

and an upper bound coming from Tougeron's theorem on the sufficiency of the $(\mu(S)+1)$-jet [78]:

$$
d(S) \leq \mu(S)+1
$$

One easily observes here an asymptotically big gap between the bounds.

\subsection{Some previously known constructions}

The other side of the problem is a construction of curves with prescribed singularities. The two following ways, which are, in fact, due to classics, were tried in this direction.

The first one is to construct somehow a curve of the given degree, which is degenerate with respect to the requested curve, and then deform it in order to get the prescribed singularities. For example, Severi's condition (1.1) is obtained as follows: take the union of $d$ generic straight lines, show that all $d(d-1) / 2$ nodes of such a curve can be smoothed or preserved independently, then smooth $d(d-1) / 2-n$ nodes and get the requested irreducible curve. Nodal curves on the blown up projective plane can be constructed using the same idea [2]. Another example, a construction of curves with nodes, cusps and ordinary triple points, was considered in [23]. Note that the number of cusps and triple points in the latter case was bounded from above by $2 d / 3$, whereas all known restrictions, in particular (1.2), bound the number of arbitrary singularities by a quadratic function of $d$. Natural difficulties, appearing this way, do not allow, actually, to extend the range of application. Namely, we have to know deformations of singularities of the initial degenerate curve, and that the needed deformations can be realized simultaneously in the space of curves of a given degree.

The second way consists in a construction especially adapted to the given degree and given collection of singularities. It may be based on a sequence of rational transformations of the plane applied to a more or less simple initial curve in order to get a curve of a given 
degree with given singularities. Or it may consist in an invention of a polynomial defining the required curve. This can be illustrated by constructions of singular curves of degrees $\leq 6$, cited above. The constructions of cuspidal curves in [40], [44], [93] fall in the same class. Namely, Ivinskis [44] constructs curves of degree $d=6 q$ with $9 q^{2}=d^{2} / 4$ cusps from a curve $F_{6}\left(x_{0}, x_{1}, x_{2}\right)=0$ of degree 6 with 9 cusps, by a $q^{2}$-sheeted branched covering of the plane:

$$
F_{6}\left(x_{0}^{q}, x_{1}^{q}, x_{2}^{q}\right)=0
$$

Hirano [40] improves Ivinskis' procedure and obtained, in particular, curves of degree $d=2 \cdot 3^{k}$ with $9\left(9^{k}-1\right) / 8$ cusps. At last, Zariski-type constructions (see [93], chapter 8 , and [76])

$$
F_{2 q}^{3}+G_{3 q}^{2}=0
$$

where $F_{2 q}=0, G_{3 q}=0$ are generic curves of degrees $2 q, 3 q$, respectively, give a curve of degree $d=6 q$ with $6 q^{2}=d^{2} / 6$ cusps. This approach does, however, not lead to any general answer because of the following reasons. First, for any collection of singularities we have to invent a new construction, and second, obtaining a curve with a certain number of singularities, we cannot guarantee that there are curves with smaller numbers of these singularities; for instance, in the above constructions of cuspidal curves, the cusps are dependent, which does not allow to smooth them independently.

Another idea, based on a modification of the Viro method of gluing polynomials (see the original method in [84], [85], [86]) and on the independence of singular point deformations, was suggested in [67], [43]. It was shown, for instance, that if

$$
n+2 k \leq \frac{d^{2}}{2}+O(d)
$$

then there exists an irreducible curve of degree $d$ with exactly $n$ nodes and $k$ cusps. Below we describe some details of the procedure but here we only say that it requires, first, to have initial curves of small degrees having given singularities, and second, to check that the ESF of curves of these small degrees are smooth and have "regular" dimensions. Such requirements are quite restrictive, but, as far as we have constructed a singular curve, we can remove any singularities keeping the others, i.e. any smaller collection of singularities is realizable as well. One more advantage is that the procedure works over the reals.

Finally, we emphasize the common defect of all these constructions: they are not applicable to the problem of finding a normal form of a single singularity.

\subsection{Asymptotically optimal sufficient existence conditions}

Here we describe a new approach to the problem on the existence of curves of a given degree with given topological singularities, that gives an asymptotically optimal sufficient existence condition for arbitrary singularities [28]. The case of analytic singularities remains still open, we discuss it in the last section.

Theorem 1 If an integer $d>0$ and topological singularities $S_{1}, \ldots, S_{r}$ satisfy

$$
\sum_{i=1}^{r} \mu\left(S_{i}\right) \leq \frac{d^{2}}{392},
$$

then there exists an irreducible curve of degree $d$ with $r$ singular points of types $S_{1}, \ldots, S_{r}$ as its only singularities. 
Theorem 2 For any topological singularity $S$ there exists an irreducible curve of degree

$$
d \leq 14 \sqrt{\mu(S)}
$$

having a singular point of type $S$ as its only singularity.

These conditions are optimal with respect to the exponents of $d$ and $\mu(S)$, as compared with the necessary conditions (1.2), (1.3).

The proof is done in three main steps:

Step 1. Existence of a curve of degree $d$ with a singular point of the given topological type $S$ is reduced to a relation

$$
h^{1}\left(\mathbb{P}^{2}, \mathcal{J}_{X}(d)\right)=0
$$

for the ideal sheaf $\mathcal{J}_{X} \subset \mathcal{O}_{\mathbb{P}^{2}}$ of a certain zero-dimensional subscheme $X \subset \mathbb{P}^{2}$, associated with $S$, such that

$$
\operatorname{deg} X \leq a \cdot \mu(S), \quad a=\text { const }>0 .
$$

Step 2. Given a scheme $X \in \mathcal{G S}$ (cf. Section 1.5), it may happen that

$$
h^{1}\left(\mathbb{P}^{2}, \mathcal{J}_{X}(d)\right)>0
$$

for $d \sim \operatorname{deg} X$. Due to the semi-continuity of $h^{1}$ we reduce this parameter by choosing a generic element $X_{0}$ in the corresponding Hilbert scheme. In addition, we show that

$$
h^{1}\left(\mathbb{P}^{2}, \mathcal{J}_{X_{0}}(d)\right)=0 \quad \text { as } \quad d \geq b \sqrt{\operatorname{deg} X_{0}}, \quad b=\text { const }>0 .
$$

Step 3. The final stage is a construction of curves with many singular points. This is done by means of a version of the Viro method.

Below we describe each step in more detail.

\subsection{Zero-dimensional schemes associated with topological singu- larities}

Let $z$ be a point of a reduced curve $C \subset \mathbb{P}^{2}$. It is called essential if $C$ is singular at $z$. If $q \neq z$ is infinitely near to $z$, we denote by $C_{(q)}$ (respectively, $\widehat{C}_{(q)}$ ) the strict (respectively, total) transform under the composition of blowing-ups, $\pi_{(q)}: S_{(q)} \rightarrow S$ defining $q$. We call $q$ essential if it is not a node (ordinary double point) of the union of $C_{(q)}$ with the reduced exceptional divisor.

For $z \in C$ let $T(C, z)$ denote the (infinite) complete embedded resolution tree of $(C, z)$ with vertices the points infinitely near to $z$. It is naturally oriented, inducing a partial ordering on its vertices such that $z<q$ for all $q \in T(C, z) \backslash\{z\}$. If $z \notin C$ we define $T(C, z)$ to be the empty tree. Moreover, let

$$
T^{*}(C, z):=\{q \in T(C, z) \mid q \text { is essential }\}
$$

denote the tree of essential points of $(C, z)$ which is a finite subtree of $T(C, z)$.

Let $T^{*} \subset T(C, z)$ be a finite, connected tree, containing $T^{*}(C, z)$. For any point $q \in T^{*}$ and any $f \in \mathcal{O}_{S, z}$ denote by $f_{(q)}$, respectively $\hat{f}_{(q)}$, the strict, respectively total, transform 
under the modification $\pi_{(q)}$ defining $q$. Put $m_{q}:=\operatorname{mt}\left(C_{(q)}, q\right), \hat{m}_{q}:=\operatorname{mt}\left(\widehat{C}_{(q)}, q\right)$ and define the ideal

$$
J:=J\left(C, T^{*}\right):=\left\{f \in \mathcal{O}_{\mathbb{P}^{2}, z} \mid \operatorname{mt}\left(\hat{f}_{(q)}, q\right) \geq \hat{m}_{q}, \quad q \in T^{*}\right\} \subset \mathcal{O}_{\mathbb{P}^{2}, z}
$$

and the subscheme of $S$ defined by $J$,

$$
X:=X\left(C, T^{*}\right)=Z(J), \quad \mathcal{O}_{X, z}:=\mathcal{O}_{\mathbb{P}^{2}, z} / J,
$$

which is concentrated on $\{z\}$. The scheme $X$ is called a generalized singularity scheme and the class of zero-dimensional subschemes of $\mathbb{P}^{2}$, constructed in this way, is denoted by $\mathcal{G S}$. The subclass of schemes $X \in \mathcal{G S}$ with $T^{*}=T^{*}(C, z)$ is denoted by $\mathcal{S}, X \in \mathcal{S}$ is called a singularity scheme.

A scheme $X \in \mathcal{G S}$ defines uniquely a topological singularity, and almost all germs $f \in J$, where $J \subset \mathcal{O}_{\mathbb{P}^{2}, z}$ is the ideal of $X$, belong to this topological type.

The following statement reduces the existence problem for one singularity to an $h^{1}$ vanishing for ideal sheaves of zero-dimensional schemes in the plane. Let $(C, z)$ be a reduced germ of an algebroid curve, $L$ be a generic straight line (a smooth germ) through $z$, and let the scheme $X \in \mathcal{G S}$ be defined by the germ $(C L, z)$ and the tree, which contains $T^{*}(C, z)$ and the first non-essential point of any local branch of $(C, z)$ (by abuse of notation, $C$ denotes the curve as well as an equation for it).

\section{Lemma 1 If}

$$
h^{1}\left(\mathbb{P}^{2}, \mathcal{J}_{X}(d-1)\right)=0,
$$

where $\mathcal{J}_{X} \subset \mathcal{O}_{\mathbb{P}^{2}}$ is the ideal sheaf of $X$, then there exists an irreducible curve of degree $d$ having only one singular point, and this point is topologically equivalent to $(C, z)$.

We illustrate the proof in the case of an ordinary singularity of multiplicity $m$ (represented by a transversal intersection point of $m$ smooth branches). We take $X^{\prime} \in \mathcal{G S}, X^{\prime} \subset X$, concentrated at the point $z$ and defined by the ideal $\mathfrak{m}_{z}^{m+1} \subset \mathcal{O}_{\mathbb{P}^{2}, z}$. The relation

$$
h^{1}\left(\mathbb{P}^{2}, \mathcal{J}_{X^{\prime}}(d-1)\right)=0
$$

means that the linear conditions defining $X^{\prime}$, i.e. the vanishing of the $m$-jet of the Taylor series at $z$, are independent in the space of curves of degree $d-1$. Hence there exists a curve $C$ of degree $d-1$ whose $m$-jet at $z$ is a non-degenerate $m$-form, that determines the given singularity at $z$. Since $C$ may not be irreducible with many singularities, we consider $C L$ and use a similar reasoning to show for each singular point $z_{i} \neq z$ of $C L$ the existence of a curve $C_{i}$ of degree $d$ having the given singularity at $z$ but not $z_{i}$. Finally, using Bertini's theorem, a generic element of the linear system $\lambda C L+\sum \lambda_{i} C_{i}$ gives the required irreducible curve with $z$ as its only singular point.

\section{6 $h^{1}$-vanishing criteria for zero-dimensional schemes in the plane}

One can easily show that $X$ and $d$ in Lemma 1 satisfy (1.6). Hence, for the asymptotically optimal results (1.4), (1.5), one has to prove (1.8) under condition (1.7).

Given a scheme $X \in \mathcal{G S}$, it may happen that

$$
h^{1}\left(\mathbb{P}^{2}, \mathcal{J}_{X}(d)\right)>0
$$


for $d \sim \operatorname{deg} X$. Due to the semi-continuity of $h^{1}$ we reduce this parameter by choosing a generic element in the corresponding Hilbert scheme $\mathcal{H}(X)$. Namely we show that $\mathcal{H}(X)$ is irreducible, and any two elements $X_{0}, X_{1} \in \mathcal{H}(X)$ can be connected by a family $X_{t} \in \mathcal{H}(X)$, $t \in[0,1]$, with underlying families of topologically equivalent germs $\left(C_{t}, z_{t}\right)$ and trees $T_{t} \subset$ $T\left(C_{t}, z_{t}\right), t \in[0,1]$, defining these schemes. Then we prove

Lemma 2 If $X \in \mathcal{G S}$ is defined by a germ $(C, z)$ and satisfies

$$
\operatorname{deg} X<\frac{\left(d-\operatorname{mt} X-\mathrm{mt}_{s} X\right)^{2}}{10}
$$

where $\mathrm{mt} X$ is the multiplicity of $C$ at $z$ and $\mathrm{mt}_{s} X$ is the total multiplicity of the singular branches of $C$ at $z$, then there exists $X_{0} \in \mathcal{H}(X)$ such that

$$
h^{1}\left(\mathbb{P}^{2}, \mathcal{J}_{X_{0}}(d)\right)=0 .
$$

Since $\mathrm{mt}_{s} X \leq \mathrm{mt} X \leq \sqrt{\mu(X)}+1$, the estimate (1.9) can be reduced to (1.7).

To find such a generic scheme $X_{0}$, we follow Hirschowitz's ideas [41], which he applied to the same problem for schemes of generic fat points in the plane. First, a good "approximation" of a generic scheme can be obtained by requiring a general position with respect to straight lines, that means, the intersection with any straight line should be not too big. The second idea consists in using an inductive procedure based on

Lemma 3 Let $L$ be a straight line, $X \subset \mathbb{P}^{2}$ be a zero-dimensional scheme. Then

$$
h^{1}\left(\mathbb{P}^{2}, \mathcal{J}_{X: L}(d-1)\right)=0, \quad \operatorname{deg}(X \cap L) \leq d+1,
$$

(where $X: L$ is the residue of $X$ with respect to $L$, and $X \cap L$ is the scheme-theoretic intersection) implies

$$
h^{1}\left(\mathbb{P}^{2}, \mathcal{J}_{X}(d)\right)=0
$$

In our case we perform the same procedure, using the main observation that the class $\mathcal{G S}$ is closed with respect to the residue operation (which does not hold for the more natural subclass $\mathcal{S})$ :

Lemma 4 In the previous notation, if $X \in \mathcal{G S}$ then $X: L \in \mathcal{G S}$.

\subsection{Gluing singular points}

Given topological singularities $S_{1}, \ldots, S_{r}$ and an integer $d>0$, we want to construct an irreducible curve of degree $d$ with exactly $r$ singular points of types $S_{1}, \ldots, S_{r}$, respectively.

First, with any $S_{i}, 1 \leq i \leq r$, we associate the zero-dimensional scheme $X_{i}$ as in Lemma 1 , an integer $d_{i}$ such that

$$
h^{1}\left(\mathbb{P}^{2}, \mathcal{J}_{X_{i}}\left(d_{i}-1\right)\right)=0, \quad i=1, \ldots, r,
$$

and an irreducible curve $C_{i}$ of degree $d_{i}$, having one singular point of type $S_{i}$. Then we take a zero-dimensional scheme $Y \subset \mathbb{P}^{2}$ of $r$ generic fat points of multiplicities $d_{1}+1, \ldots, d_{r}+1$, satisfying

$$
h^{1}\left(\mathbb{P}^{2}, \mathcal{J}_{Y}(d)\right)=0
$$


By Hirschowitz's theorem [41], the latter relation holds if

$$
\sum_{i=1}^{r} \frac{\left(d_{i}+1\right)\left(d_{i}+2\right)}{2}<\left[\frac{(d+3)^{2}}{4}\right]
$$

Finally, we take a curve $C \in\left|\mathcal{J}_{Y}(d)\right|$ and simultaneously deform its singular points in order to produce a curve with singularities $S_{1}, \ldots, S_{r}$. Here we use a Viro-type one-parametric deformation, which looks as if we replace the zero $d_{i}$-jet of the curve $C$ at a point $z_{i} \in Y$ by an equation of a curve $\widetilde{C}_{i}$ of degree $d_{i}$, close to $C_{i}$ and belonging to the same ESF.

\section{Smoothness of equisingular and equianalytic families}

In this section we discuss results on the smoothness of the variety of projective curves on a smooth surface $\Sigma$ with fixed singularity type, consi dered as a subscheme of the Hilbert scheme of $\Sigma$. We actually consider a stronger condition, the so-called $T$-property, which states that the conditions imposed by the singularities are independent (or transversal), which is more natural than just smoothness.

Starting with methods and results for arbitrary surfaces, we concentrate on the most important case $\Sigma=\mathbb{P}^{2}$, and then finish with a few remarks about rational and $K 3$-surfaces and surfaces of general type.

\section{$2.1 \quad T$-property}

Let $\Sigma$ be a smooth projective surface (embedded in some projective space) and let Hilb ${ }_{h}^{\Sigma}$ the Hilbert scheme of $\Sigma$, parametrizing closed subschemes of $\Sigma$ with given Hilbert polynomial $h$. Let $V=V_{h}\left(S_{1}, \ldots, S_{r}\right)$ denote the locally closed subscheme of reduced curves on $\Sigma$, having exactly $r$ singularities of (analytic or topological) types $S_{1}, \ldots, S_{r}$ (cf. [87] for nodes and cusps and [26], [27] in general for the existence of $V$ ). Intuitively, each singularity imposes open and closed conditions and all conditions for all singular points define a certain locally closed subvariety of $\operatorname{Hilb}_{h}^{\Sigma}$ and we say that $V$ has the $T$-property if all closed conditions for all singular points are independent, that is, the corresponding subvarieties of $\mathrm{Hilb}_{h}^{\Sigma}$ are smooth hypersurfaces and intersect transversally. Hence, $V$ is then smooth of the "expected codimension", which is equal to the number of closed conditions.

Already the Italian geometers [61], [59], [60] noticed that it is possible to express the $T$-property infinitesimally for families of plane curves with nodes and cusps. The classical term for $T$-property of such families is "completeness of the linear characteristic series of families with nodes and cusps" (cf. [93], appendix to Chapter V). Best known is certainly Severi's result [61] saying that nodal curves in $\mathbb{P}^{2}$ satisfy the $T$-property.

To give a precise definition, recall that $H^{0}\left(C, \mathcal{N}_{C / \Sigma}\right)$ is the Zariski tangent space to Hilb $_{h}^{\Sigma}$ at $C$, as well as the space of first order embedded deformations of $C$ in $\Sigma$, where $\mathcal{N}_{C / \Sigma}=\mathcal{O}_{\Sigma}(C) \otimes \mathcal{O}_{C}$ is the normal sheaf of $C$ in $\Sigma$. It appears in the exact sequence

$$
0 \rightarrow \Theta_{C} \rightarrow \Theta_{\Sigma} \otimes_{\mathcal{O}_{\Sigma}} \mathcal{O}_{C} \rightarrow \mathcal{N}_{C / \Sigma} \rightarrow \mathcal{T}_{C}^{1} \rightarrow 0
$$

where $\mathcal{T}_{C}^{1}$ is a skyscraper sheaf concentrated on the singularities of $C$ with $\mathcal{T}_{C, z}^{1} \cong \mathcal{O}_{\Sigma, z} / I_{z}^{e a}$ and

$$
I_{z}^{e a}=\left(f, f_{x}, f_{y}\right) \mathcal{O}_{\Sigma, z}
$$


for $f(x, y)=0$ a local equation of $C$ at $z$. Moreover, we have Wahl's equisingularity ideal [88],

$$
I_{z}^{e s}=\left\{g \in \mathcal{O}_{\Sigma, z} \mid f+\varepsilon g \text { is equisingular over } \operatorname{Spec}\left(\mathbb{C}[\varepsilon] / \varepsilon^{2}\right)\right\}
$$

and inclusions

$$
I_{z}^{e a} \subset I_{z}^{e s}
$$

Let $J^{e a}, J^{e s}$ be the corresponding ideal sheaves defining 0-dimensional schemes $X^{e a} \supset X^{e s}$ on $\Sigma$. Note that $X^{e a}$ and $X^{e s}$ are actually subschemes of $C$. For simplicity we use the superscript 1 and mean ea (respectively es) if we consider families of curves with fixed analytic (respectively topological) singularity types $S_{1}, \ldots, S_{r}$. To be consistent in notations we sometimes write $V^{\prime}=V_{h}^{\prime}\left(S_{1}, \ldots, S_{r}\right)$ for $V=V_{h}\left(S_{1}, \ldots, S_{r}\right)$.

We set

$$
J_{X^{\prime} / C}(C):=\left.J^{\prime} \mathcal{O}_{C}(C)\right|_{C}=\left.\operatorname{Ker}\left(\mathcal{O}_{\Sigma}(C) \otimes \mathcal{O}_{C} \rightarrow \mathcal{O}_{X^{\prime}}\right)\right|_{C}
$$

and then, from (2.1) we deduce the exact sequence (noting that $\mathcal{O}_{X^{e a}}$ surjects onto $\mathcal{O}_{X^{e s}}$ )

$$
0 \rightarrow \mathcal{J}_{X^{\prime} / C}(C) \rightarrow \mathcal{O}_{\Sigma}(C) \otimes \mathcal{O}_{C} \rightarrow \mathcal{O}_{X^{\prime}} \rightarrow 0
$$

It follows from general deformation theory (cf. [26], [27]) that

- $H^{0}\left(C, J_{X^{\prime} / C}(C)\right)=$ Zariski tangent space to $V^{\prime}$ at $C$

- $H^{1}\left(C, J_{X^{\prime} / C}(C)\right)=0 \Leftrightarrow V^{\prime}$ is smooth at $C$ of the expected dimension $C^{2}+1-p_{a}(C)-$ $\operatorname{deg}\left(X^{\prime}\right)$.

Hence, we may define the $T$-property of $V^{\prime}$ by the vanishing of $H^{1}\left(C, J_{X^{\prime} / C}(C)\right)$ for all $C$ in $V^{\prime}$. This holds, of course, for an empty $V^{\prime}$, hence, $T$-property has nothing to do with existence.

We remark that the expected codimensions are

$$
\begin{aligned}
& \operatorname{deg}\left(X^{e a}\right)=\tau(C), \quad \text { respectively } \\
& \operatorname{deg}\left(X^{e s}\right)=\tau(C)-\bmod (C),
\end{aligned}
$$

where

$$
\tau(C)=\sum_{x \in C} \operatorname{dim}_{\mathbb{C}} \mathcal{T}_{C, x}^{1}
$$

is the total Tjurina number and

$$
\bmod (C)=\sum_{x \in C} \bmod (C, x)
$$

the total modality in the sense of Arnold, that is, with respect to right equivalence ([3]).

Note that fixing the Hilbert polynomial of $C$ is equivalent to fixing the arithmetic genus $p_{a}(C)$ and, either the degree of the embedding or the self-intersection $C^{2}$ or the intersection with the canonical divisor, $C \cdot K_{\Sigma}$. Classically, instead of Hilb ${ }_{h}^{\Sigma}$, the complete linear system $|C|$ on $\Sigma$ is considered. By the remark above, the incidence variety is flat over $|C|$ and hence, there exists a unique (injective) morphism $|C| \rightarrow \operatorname{Hilb}_{h(C)}^{\Sigma}$, which, on the tangent level, corresponds to

$$
H^{0}\left(\Sigma, \mathcal{O}_{\Sigma}(C)\right) / H^{0}\left(\Sigma, \mathcal{O}_{\Sigma}\right) \hookrightarrow H^{0}\left(\Sigma, \mathcal{O}_{\Sigma}(C) \otimes \mathcal{O}_{C}\right)
$$

Hence, we may consider $|C|$ as a subscheme of Hilb. If $\Sigma$ is a regular surface $\left(h^{1}\left(\mathcal{O}_{\Sigma}\right)=0\right)$, then $|C|$ coincides with Hilb. 


\subsection{Curves on general surfaces}

Basically, only one sufficient condition for smoothness for general surfaces $\Sigma$ is known. The following result is proven in [76] for curves with nodes and cusps and in [26], respectively [27], for arbitrary analytic, respectively topological, singularity types and gives the following condition for $T$-property.

If $C$ is irreducible then $H^{1}\left(C, J_{X^{\prime} / C}(C)\right)=0$ if

$$
-K_{\Sigma} C>\operatorname{deg} X^{\prime}-\varepsilon^{\prime}
$$

where $\varepsilon^{\prime} \geq 0$ is the "isomorphism defect" (cf. [26], [27]).

If $C$ is reducible and $C=C_{1} \cup \cdots \cup C_{s}$, the decomposition into irreducible components, then $H^{1}\left(C, J_{X^{\prime} / C}(C)\right)=0$ if for $i=1, \ldots, s$

$$
-K_{\Sigma} C_{i}>\operatorname{deg}\left(X^{\prime} \otimes \mathcal{O}_{C_{i}}\right)-\varepsilon_{i}^{\prime}
$$

where $\varepsilon_{i}^{\prime} \geq 0$ is a local invariant with $\varepsilon_{i}^{e a} \geq 1$ and $\operatorname{deg}\left(X^{e a} \otimes \mathcal{O}_{C_{i}}\right)=\Sigma_{j \neq i} C_{j} C_{i}+\tau\left(C_{i}\right)$.

Since nodes do not contribute to the right-hand side of (2.3) and (2.4) we obtain that the variety of nodal curves on $\Sigma$ with a fixed number of nodes has the $T$-property at $C$ if $-K_{\Sigma} C_{i}>0$ for all irreducible components $C_{i}$ of $C$.

(2.3) and (2.4) may be considered as the "standard" inequalities, except for the isomorphism defects $\varepsilon^{\prime}$ and $\varepsilon_{i}^{\prime}$.

These local isomorphism defects come from a refinement of the Riemann-Roch formula for not locally free rank 1 sheaves (cf. [26]) and can be quite big for $J_{X^{e s} / C}$. For example,

$$
\varepsilon^{e s}=\frac{m(m-1)}{2}-2
$$

for an ordinary $m$-tuple point, $m \geq 3$ (cf. [27]).

As a result we obtain, if $S_{1}, \ldots, S_{r}$ are topological types of ordinary singularities, that $V_{h}^{e s}\left(S_{1}, \ldots, S_{r}\right)$ has the $T$-property at $C=C_{1} \cup \cdots \cup C_{s}$ if

$$
-K_{\Sigma} C_{i}>\sum_{\substack{x \in C_{i} \cap \operatorname{Sing}(C) \\ \operatorname{mt}_{x}(C)>2}} \operatorname{mt}_{x}\left(C_{i}\right), \quad i=1, \ldots, s,
$$

where $\mathrm{mt}_{x}$ denotes the multiplicity at $x$. For $\Sigma=\mathbb{P}^{2}$, the left-hand side is equal to $3 \cdot \operatorname{deg}\left(C_{i}\right)$ and we obtain a result of Giacinti-Diebolt [22].

The vanishing of $H^{1}\left(C, J_{X^{\prime} / C}(C)\right)$ implies $H^{1}\left(C, \mathcal{O}_{\Sigma}(C) \otimes \mathcal{O}_{C}\right)=0$ and hence, the smoothness of $\operatorname{Hilb}_{h}^{\Sigma}$ at $C$. But it has another important consequence:

If $X^{\prime}=X^{e a}$ then $H^{0}\left(C, \mathcal{O}_{\Sigma}(C) \otimes \mathcal{O}_{C}\right) \rightarrow H^{0}\left(C, \mathcal{T}_{C}^{1}\right)$ is surjective and any infinitesimal deformation of the singularities $S_{1}, \ldots S_{r}$ can be lifted to an infinitesimal deformation of the global curve $C$ as a subscheme of $\Sigma$. Moreover, since $H^{1}\left(C, \mathcal{O}_{\Sigma}(C) \otimes \mathcal{O}_{C}\right)=0$ these deformations are unobstructed and hence, any deformation of $S_{1}, \ldots, S_{r}$ is induced by a subfamily of curves in $\Sigma$ close to $C$. We call this the independence of deformations of $S_{1}, \ldots, S_{r}$ (or regular adjacency) in $\mathrm{Hilb}_{h}^{\Sigma}$.

More generally, let $\left\{S_{1}, \ldots, S_{t}\right\}$ be analytic and $\left\{S_{t+1}, \ldots, S_{r}\right\}$ topological singularity types and let $X^{\prime}$ denote the corresponding mixed analytic-topological scheme. Then the vanishing of $H^{1}\left(C ; J_{X^{\prime} / C}(C)\right)$ implies the independence of deformations of $S_{1}, \ldots, S_{t}$ without changing the topological type of $S_{t+1}, \ldots, S_{r}$. 


\subsection{Curves in $\mathbb{P}^{2}$}

Severi's result [61], that the family of plane curves of degree $d$ having a fixed number of nodes is a $T$-variety, does not generalize to more complicated singularities. Wahl [87] showed the existences of an ESF of curves of degree 104 with 900 ordinary cusps and 3636 nodes without $T$-property; the reduction of his family is, however, smooth (of dimension bigger than the expected one).

Luengo constructed in [52] an irreducible plane curve of degree 9 having one singularity of type $A_{35}$ such that the corresponding ESF has the expected dimension but is not smooth, not even its reduction. Further examples were found in [71].

The classical result [93] is that the family $V_{d}(n, k)$ of curves of degree $d$ with $n$ nodes and $k$ cusps is a $T$-variety at an irreducible curve $C$ if

$$
k<3 d .
$$

For $\mathbb{P}^{2}$ and arbitrary singularity types $S_{1}, \ldots, S_{r}$ (analytic or topological), the general approach from Section 2.2 gives that $V_{d}\left(S_{1}, \ldots, S_{r}\right)$ has the $T$-property at an irreducible curve $C$, if

$$
\operatorname{deg}\left(X^{\prime}\right)-\varepsilon^{\prime}<3 d
$$

(cf. [26], [27]) which is of the same nature. Indeed we cannot improve this inequality substantially if we consider $X^{\prime}$ as a subscheme of the given curve $C$ and use vanishing theorems on $C$.

Progress has been made by considering $X^{\prime}$ not as a subscheme of $C$ but as a subscheme of $\mathbb{P}^{2}$ and looking for an irreducible curve $C^{\prime}$ of degree $d^{\prime}<d$ containing $X^{\prime}$ as a subscheme and then use vanishing theorems on $C^{\prime}$. This idea appears for the first time in [63] with $C^{\prime}$ a generic polar of $C$, which is irreducible if $C$ is not the union of lines through one point. This led to the sufficient condition (cf. [63], [27])

$$
\operatorname{deg} X^{\prime}-\tilde{\varepsilon}^{\prime}<4 d-4
$$

where $\tilde{\varepsilon}^{\prime} \geq 0$, which can be computed or estimated by local methods (cf. [27]). Note that $C$ needs not to be irreducible.

The use of $C^{\prime}$ instead of $C$ is possible, since for $\mathbb{P}^{2}$ we have

$$
h^{1}\left(\mathbb{P}^{2}, \mathcal{O}_{\mathbb{P}^{2}}(k)\right)=h^{2}\left(\mathbb{P}^{2}, \mathcal{O}_{\mathbb{P}^{2}}(k)\right)=0(k \geq 0)
$$

hence $H^{1}\left(C, J_{X^{\prime} / C}(C)\right)=0 \Leftrightarrow H^{1}\left(\mathbb{P}^{2}, J_{X^{\prime}}(C)\right)=0 \Leftrightarrow H^{1}\left(C^{\prime}, J_{X^{\prime} / C^{\prime}}(C)\right)=0$ for $C^{\prime}$ a curve of degree $d^{\prime} \leq d=\operatorname{deg} C$.

The above inequalities (2.6), (2.7), (2.8) have a right-hand side which is linear in $d$ while the left-hand side may be quadratic in $d$ (for example, for $d$ lines through one point). Other generalizations of the same kind were obtained in [66], [83].

In [68] and [70] the T-property (and irreducibility) was shown when the total Milnor number was bounded by a quadratic function in $d$. These bounds have been improved in [71], using ideas from [28], stating that $V_{d}\left(S_{1}, \ldots, S_{r}\right)$ has the $T$-property at $C$ and is of codimension $\sum_{i=1}^{r} \tau^{\prime}\left(S_{i}\right)$, if

$$
\sum_{i=1}^{r}\left(\mu\left(S_{i}\right)+2\right)^{4} \leq(d+6)^{2}
$$


where $\mu$ is the Milnor number, $\tau^{\prime}=\tau$ (the Tjurina number) for an analytic type and $\tau^{\prime}=\tau$ mod for a topological type. For curves with $n$ nodes and $k$ cusps $V_{d}(n, k)$ is a $T$-variety at an irreducible $C$ if

$$
\frac{4}{5} n+2 k \leq \frac{(d+6)^{2}}{18}
$$

Moreover, in [71] it was shown that the variety of curves of degree $2 p q$ having $q^{2}$ singularities of type $A_{6 p-1}$ has a non-smooth irreducible component if $p \geq 5, q \geq 3$ (cf. also Section $4)$.

\subsection{Curves on special surfaces}

Besides $\mathbb{P}^{2}$, few special surfaces have been considered where the results are better than for the general case discussed in 2.2.

So far, only nodal curves on rational surfaces, $K 3$-surfaces and surfaces of general type have been considered (cf. [74], [75], [53], [29], [14]).

Let $\Sigma$ be a smooth surface, $D$ a divisor of $\Sigma$ and $V(D, n)$ the variety of nodal curves in the complete linear system $|D|$ with exactly $n$ nodes. Tannenbaum [74] derives for a rational surface $\Sigma$ the condition

$$
-K_{\Sigma} \cdot C_{i}>0, \quad i=1, \ldots, s,
$$

for the $T$-property of $V(D, n)$ at $C=C_{1} \cup \cdots \cup C_{s}, C_{i}$ irreducible, if the generic member of $|D|$ is irreducible. Note that for a rational surface the result follows directly from (2.4) of Section 2.2.

However, for $\Sigma=\mathbb{P}_{m}^{2}$, the projective plane blown-up in $m$ generic points $p_{1}, \ldots, p_{m}$, we have better results which are for large $m$ asymptotically (in $d$ ) nearly optimal. Let $E_{0} \subset \Sigma$ be the preimage of a generic straight line in $\mathbb{P}^{2}$ and $E_{1}, \ldots, E_{m}$ the exceptional divisors. Then, for an irreducible nodal curve $C \in\left|d E_{0}-d_{1} E_{1}-\ldots-d_{m} E_{m}\right|,(2.11)$ reads

$$
3 d>\sum_{i=1}^{m} d_{i}
$$

This gives already that $V=V\left(d ; d_{1}, \ldots, d_{m} ; n\right)$, the variety of nodal curves in $\mid d E_{0}-$ $d_{1} E_{1}-\ldots-d_{m} E_{m} \mid$ with $n$ nodes, is smooth at $C$ for $m \leq 9$. For $m \geq 10$ it is shown in [29] that $V$ has the $T$-property at $C$ if the following two conditions are fulfilled:

$$
\begin{aligned}
& {[\sqrt{2 n}]<\frac{d}{2}+3-\frac{\sqrt{2}}{2} \sqrt{\sum_{i=1}^{m}\left(d_{i}+2\right)^{2}}} \\
& {[\sqrt{2 n}]<d+3-\sqrt{2} \sqrt{2+\sum_{i=1}^{m}\left(d_{i}+2\right)\left(d_{i}+1\right)} .}
\end{aligned}
$$

If $C$ is reducible, the $T$-property holds at $C$ if analogous conditions are fulfilled for each component of $C$.

For a $K 3$-surface, $K_{\Sigma}$ is trivial, hence the above condition is never fulfilled. Tannenbaum shows in [75] that the $T$-property holds for $V(D, n)$ at $C \in V(D, n)$ if and only if $C$ is 
irreducible. Also, the independence of deformations of the nodes fails if $C$ is reducible. Nevertheless, $V(D, n)$ is always smooth at $C=C_{1} \cup \cdots \cup C_{s}$ of dimension $\operatorname{dim}|D|-n+(s-1)$.

In [14] Chiantini and Sernesi consider nodal curves $C$ on surfaces of general type $\Sigma$. They study a rank 2 bundle on $\Sigma$ associated to the nodes of $C$, and use an inequality for the Bogomolov instability of this bundle, in order to prove that $V(C, n)$ has the $T$-property at an irreducible $C$. Namely, if $C$ is numerically equivalent to $p K_{\Sigma}, p \in \mathbb{Q}, p>2$, then $V(D, n)$ has the $T$-property, provided

$$
n<\frac{1}{4} p(p-2)\left(K_{\Sigma}\right)^{2}
$$

or if

$$
n<\frac{1}{4}(p-1)^{2}\left(K_{\Sigma}\right)^{2}
$$

$p$ odd, and the Neron-Severi group is $N S(\Sigma)=\mathbb{Z} \cdot K_{\Sigma}$. These results seem to be asymptotically nearly optimal (see also Section 4 ).

\section{$3 \quad$ Irreducibility of equisingular families}

The question about the irreducibility of ESF is more delicate than the existence and smoothness problems, especially concerning sufficient conditions. Rather difficult from the algebraicgeometric point of view, it is of special topological interest, being connected with the problem on the fundamental group of the complement of a plane complex algebraic curve [93] (and through this, with the classification of complex algebraic surfaces). Even the case of plane nodal curves appeared to be very hard: Severi started to handle it [61], but failed (see details in [2], [39]), then Arbarello and Cornalba [2] made some progress, until Harris [39] proved that the family $V_{d}(n)$ of irreducible plane curves of degree $d$ with $n$ nodes as their only singularities is irreducible for any

$$
n \leq \frac{(d-1)(d-2)}{2}
$$

The classical Zariski examples of reducible ESF and other such examples [22], [68], show that for more complicated singularities, beginning with ordinary cusps, possible numerical sufficient conditions for the irreducibility of ESF should be different from the necessary existence conditions for curves with a given collection of singularities.

The results in this direction concern basically plane curves and we will pay the most attention to this case.

\subsection{Plane curves}

There are three main approaches to the problem about irreducibility of ESF.

(1) One possible approach consists in building a connecting path between two equisingular curves, using explicitly equations of the curves, or on projective transformations. This method works for small degrees only. Besides the classical case of conics and cubics, it is proven that all ESF of quartic curves [11] and of quintic curves [16], [90] are irreducible. For 
degrees $>5$ this is not true, and the method is no more efficient, except some very special cases [79], [80], [81].

(2) Another approach, suggested by Arbarello and Cornalba [2], consists in relating an ESF to the moduli space of curves of a given genus, known to be irreducible. This gave some particular results on families of plane nodal curves [2], and plane curves with nodes and cusps. Namely Kang [45] proved that the family $V_{d}(n, k)$ of plane irreducible curves of degree $d$ with $n$ nodes and $k$ cusps as their only singularities is irreducible if

$$
k \leq \frac{d+1}{2}, \quad \frac{(d-1)(d-2)}{2} \geq n \geq \frac{d^{2}-4 d+1}{2} .
$$

(3) The new idea by Harris [39] completed the case of plane nodal curves. It consists in proceeding inductively from rational nodal curves (whose family is classically known to be irreducible) via study of a degeneration of nodal curves to any family of curves of a given degree with a given number of nodes. Further development of this idea lead to new results by Ran [55]: an ESF of plane irreducible curves of a given degree with a given number of nodes and one ordinary singularity is irreducible, and by Kang [46]: an ESF of irreducible curves of a given degree with a given number of nodes and a given number $k \leq 3$ of cusps is irreducible. Note that the requirement to study all possible deformations of curves considered does not allow to extend such an approach to more complicated singularities or to a large number of any singularities different from nodes.

(4) The following approach is applicable to ESF of curves (and even projective hypersurfaces of any dimension) with any quantities of arbitrary singularities and provides the most general results. So we explain it in more detail. Namely, let $V$ be an ESF of plane curves of degree $d$ with a given collection of (topological or analytic) singularities. With an isolated singular point $z$ of a plane curve $C$ we can associate zero-dimensional schemes $X^{s}(z)$ and $X^{a}(z)$ such that

- for almost all elements $f$ in the local ideal $J_{X^{s}(z)} \subset \mathcal{O}_{\mathbb{P}^{2}, z}$ of the scheme $X^{s}(z)$ (respectively in the local ideal $J_{X^{a}(z)} \subset \mathcal{O}_{\mathbb{P}^{2}, z}$ of the scheme $\left.X^{a}(z)\right) C$ and $C+f$ have the same topological (respectively analytic) singularity at $z$,

- $X^{s}(z)$ (respectively $X^{a}(z)$ ) varies in an irreducible flat family $\mathcal{X}(S)$ as $z$ runs through all singular points of topological (respectively analytic) type $S$.

For instance, $X^{s}(z)$ can be defined as $X\left(C, T^{*}(C, z)\right)$ in the notation of section 2.5, and $X^{a}(z)$ can be defined by the ideal

$$
J_{X^{a}(z)}=\left(\mathfrak{m}_{z}\right)^{\mu(z)+2} \subset \mathcal{O}_{\mathbb{P}^{2}, z}
$$

(see [83], Theorem 1.2.1). If a curve $C \in V$ has singularities $S_{1}, \ldots, S_{r}$, then we define a natural morphism

$$
\Phi: \widetilde{V} \rightarrow \mathcal{X}\left(S_{1}\right) \times \cdots \times \mathcal{X}\left(S_{r}\right)
$$

where $\widetilde{V}$ is a finite covering of $V$, consisting of curves $C \in V$ with ordered singular points. By the construction of $\mathcal{X}(S)$, for the irreducibility of $V$ it is enough to show that $\Phi$ is surjective and its fibers are irreducible (open sets of projective spaces). Both conditions follow from

$$
h^{1}\left(\mathbb{P}^{2}, \mathcal{J}_{X(C)}(d)\right)=0, \quad \text { for each } C \in V,
$$


where $X(C)$ is the union of schemes $X^{s}(z)$ (or $X^{a}(z)$, according as we consider topological or analytic singularities) taken over all singular points $z \in C$. The inequality, derived easily from [26], Proposition 5.2,

$$
\operatorname{deg} X(C)<3 d
$$

is sufficient for (3.1). The right-hand side here is linear in $d$, whereas the left-hand side, being the sum of certain positive invariants of $S_{1}, \ldots, S_{r}$, may be of order $d^{2}$. We observe also that (3.1) may fail for some curves $C \in V$ as (3.2) fails, even in the case of nodal curves [66]. To improve the result, one can weaken the requirement to

$$
V_{\text {reg }}=\left\{C \in V \mid h^{1}\left(\mathbb{P}^{2}, \mathcal{J}_{X(C)}(d)\right)=0\right\}
$$

is open dense in $V$. In other words

$$
\operatorname{dim}\left(V \backslash V_{\text {reg }}\right)<\operatorname{dim} V,
$$

where $\operatorname{dim} V$ is the virtual "regular" dimension of $V$ (in fact, the minimal possible actual dimension).

In [68], [69], [70] the failure of (3.1) was interpreted as: the singular points of $C \in V$ lie on a curve of a relatively small degree. Then, using an irreducible curve of degree $n<\alpha d$, passing through the scheme $X^{s}(C)$ or $X^{a}(C)$, one can estimate the dimension of the set of such curves $C$ and get the following sufficient condition for the irreducibility of $V$ :

$$
n+2 k<\frac{d^{2}}{225}
$$

if $V=V_{d}(n, k)$, and

$$
\begin{gathered}
\sum_{i=1}^{r} \mu\left(S_{i}\right) \leq \min _{1 \leq i \leq r} f\left(S_{i}\right) \cdot d^{2}, \\
f(S)=\frac{2}{(\mu(S)+\operatorname{mt}(S)-1)^{2}\left(3 \mu(S)-\operatorname{mt}(S)^{2}+3 \cdot \operatorname{mt}(S)+2\right)^{2}} .
\end{gathered}
$$

The latter conditions have a right-hand side quadratic in $d$.

D. Barkats [7] approached the problem (3.3) from the view point of the theory of zerodimensional subschemes of the plane [15] and obtained inequalities with bigger coefficients of $d^{2}$,

$$
3 n+5 k<\frac{7}{40} d^{2}-\frac{1}{8} d-5
$$

to be sufficient for the irreducibility of $V_{d}(n, k)$, and

$$
\sum_{i=1}^{r} b\left(S_{i}\right)<\frac{2 b-3}{2 b(b-1)} d^{2}-\frac{2 b-9}{2(b-1)} d
$$

to be sufficient for the irreducibility of $V$, when $S_{1}, \ldots, S_{r}$ are topological singularities, $b(S)=\operatorname{deg} X^{s}(z)$ as $z$ is of type $S$, and

$$
b=\max _{i=1, \ldots, r} b\left(S_{i}\right) .
$$




\subsection{Curves on rational surfaces}

The methods described above were extended on some rational surfaces.

So, Ran [55] showed that, for any Hirzebruch surface (fan) $\Sigma$, a family of irreducible curves of a given bidegree with a given number of nodes is irreducible. He used both, Harris' method and the theory of deformations of fans. Due to the reasons mentioned above, it is hardly possible to generalize this method to any surface and curves with arbitrary singularities.

On the other hand, the approach of [68], [69], [70] was applied to $\Sigma=\mathbb{P}_{m}^{2}$, the plane blown up at $m$ generic points, and the families $V\left(d ; d_{1}, \ldots, d_{m} ; n\right)$ of irreducible curves with $n$ nodes, belonging to the linear system $\left|d E_{0}-d_{1} E_{1}-\ldots-d_{m} E_{m}\right|$. As result, in [29] the inequalities

$$
\begin{aligned}
& {[\sqrt{2 k}]<\frac{d}{4}+1-\frac{1}{4} \sqrt{\sum_{i=1}^{m} d_{i}^{2}}} \\
& {[\sqrt{2 k}]<\frac{d}{2}+1-\frac{\sqrt{2}}{2} \sqrt{\sum_{i=1}^{m}\left(d_{i}+2\right)^{2}}}
\end{aligned}
$$

are shown to be sufficient for the irreducibility of $V\left(d ; d_{1}, \ldots, d_{m} ; n\right)$. In contrast to the irreducibility problem for plane curves, discussed in the previous subsection, here it is needed to have a certain irreducible curve going through the singularities of an arbitrary curve $C \in V=V\left(d ; d_{1}, \ldots, d_{m} ; n\right)$. But this strongly depends on the fact that only nodal curves are considered.

\section{Perspectives and problems}

In this section we indicate some problems naturally appearing in the field, expected results, and perspective ideas.

(1) The problem on the existence of plane curves of a given degree with given analytic (non-simple) singularities is still open. In general, we have only upper bounds mentioned above. The zero-dimensional scheme approach used in the topological situation does not work here, because the residue operation does not respect an analytic type of singular points. Another approach may be based on the relation between topological and analytic singularities. Let us call the quotient $C S(S)=V_{h}(S) / \sim$ (with respect to analytic equivalence) a classifying space of a topological singularity $S$. In the case of a simple singularity $S, C S(S)$ is a point, while in general it is merely a (connected) topological space (not even Hausdorff). In order to obtain a (coarse) moduli space as an algebraic variety, we have to stratify $V_{h}(S)$ into invariant strata and call the disjoint union of these strata, $M(S)$, the moduli space of a topological singularity $S$ (cf. [33]). What may be a "good" compactification of $M(S)$ ? This may be important in view of

Theorem 3 There is an open dense subset $U \subset V_{h}(S)$ such that for any analytic singularity $\mathcal{A} \in U$ there exists an irreducible curve of degree

$$
d \leq 14 \sqrt{\mu(S)}
$$

having one singular point of type $\mathcal{A}$ as its only singularity. 
We conjecture that for all topological singularities $S$, and $\mathcal{A} \in M(S)$, the minimal degree $d=d(\mathcal{A})$ such as in Theorem 3 satisfies

$$
d(\mathcal{A}) \leq a \cdot d(S)
$$

with an absolute constant $a$. This would imply an asymptotically optimal sufficient condition for the existence of curves with given analytic singularities, similar to that for topological singularities.

(2) In connection with the previous problem it seems to be perspective to develop the theory of zero-dimensional schemes in the plane of general type and of types associated with singularities, as, for instance, of class $\mathcal{G S}$. The principal problem is the computation of $h^{1}\left(\mathbb{P}^{2}, \mathcal{J}_{X}(d)\right)$ (or the so-called Castelnuovo function [15]). From our point of view it is important to estimate the minimal $d$ such that

$$
h^{1}\left(\mathbb{P}^{2}, \mathcal{J}_{X}(d)\right)=0
$$

for $X$ being a generic element in the corresponding Hilbert scheme. We conjecture that there exists an absolute constant $a>0$ such that

$$
d \geq a \sqrt{\operatorname{deg} X}
$$

provides (4.1), which would generalize Hirschowitz's theorem [41], mentioned in subsection 1.7 .

It is interesting to strengthen Hirschowitz's inequality (1.11) itself, that would improve the constants in the sufficient existence conditions involving such inequalities. In particular, in [73], (1.11) is strengthened to

$$
\max _{i<j}\left(d_{i}+d_{j}\right)<d, \quad \sum_{i=1}^{r} \frac{\left(d_{i}+1\right)\left(d_{i}+2\right)}{2}<\frac{2 d^{2}+8 d-15}{5} .
$$

The Harbourne-Hirschowitz conjecture [41], [38] states that

$$
\max _{i<j<k}\left(d_{i}+d_{j}+d_{k}\right) \leq d-3, \quad \sum_{i=1}^{r} \frac{\left(d_{i}+1\right)\left(d_{i}+2\right)}{2} \leq \frac{(d+1)(d+2)}{2}
$$

is sufficient for (1.10).

(3) In a similar way we can state the problem on the existence of hypersurfaces in $\mathbb{P}^{m}$, $m \geq 3$, of a given degree with isolated singular points of given types. Our conjecture is that the inequality

$$
\sum_{i=1}^{r} \mu\left(S_{i}\right) \leq a_{m} d^{m}
$$

with a certain positive $a_{m}$, depending only on $m$, suffices for the existence of an irreducible hypersurface of degree $d$ in $\mathbb{P}^{m}$ having $r$ isolated singular points of types $S_{1}, \ldots, S_{r}$, respectively as its only singularities. Such an estimate would be asymptotically optimal in view of Bruce's upper bounds to the total Milnor number of projective hypersurfaces [10].

We like to point out two aspects of this problem. The problem, in fact, consists of two problems - for topological singularities and for analytic ones. A possible approach to the problem may be based on the study of zero-dimensional schemes in $\mathbb{P}^{m}$. Namely, it might consist in a description of zero-dimensional schemes associated with topological or analytic 
isolated singularities, and the development of $h^{1}$-vanishing criteria for generic (in some sense) zero-dimensional schemes of certain classes, which conjecturally should look like

$$
h^{1}\left(\mathbb{P}^{m}, \mathcal{J}_{X}(d)\right)=0 \quad \text { as } \quad \operatorname{deg} X \leq a d^{m}, \quad a=\text { const }>0 .
$$

(4) In the smoothness and irreducibility problem for ESF of plane algebraic curves, the question on optimality of the numerical sufficient conditions for these properties remains still open. We recall that the sufficient condition for the existence (1.4) discussed in section 1.4 , is asymptotically optimal, i.e. it differs from the necessary condition (1.2) only in the coefficient of $d^{2}$ in the right-hand side. Another situation is observed for the smoothness and the irreducibility: there are sufficient conditions which are "linear in $d$ ", like those from [26], $[27]:$

$$
\begin{gathered}
\sum_{i=1}^{r}\left(\tau\left(S_{i}\right)-1\right)<3 d, \\
\sum_{i=1}^{r} \tau\left(S_{i}\right)<4 d-4,
\end{gathered}
$$

and ones "quadratic in $d$ ", like (3.5) in [70] and (3.6) in [8]. The latter conditions are weaker than any asymptotically optimal one, because the coefficient of $d^{2}$ in the right-hand side depends on the "worst singularity" $S_{i}$ and tends to zero as $\mu\left(S_{i}\right) \rightarrow \infty$. The following statement shows that, unlike for the existence, there cannot be an asymptotically optimal sufficient condition for the smoothness of ESF [71]:

Theorem 4 The family $V_{2 p q}\left(q^{2} \cdot A_{6 p-1}\right)$, where $A_{6 p-1}$ is the singularity given by $y^{2}+x^{6 p}=0$, has a non-smooth irreducible component if $p \geq 5, q \geq 3$.

Indeed, here we have a sequence of non-smooth ESF as the number of some singularities $S$ becomes $\sim 9 d^{2} / \mu(S)^{2}$, where $d$ and $\mu(S)$ tend to $\infty$. In Figure 1, we give a graphic illustration of the described conditions. Here $x=d^{2}$ is the squared degree of a curve, $y=\sum_{i=1}^{r} \mu\left(S_{i}\right)$ is the total complexity of the singularities. The straight lines $y=x$ and $y=x / 392$ indicate the necessary and sufficient conditions for the existence of a curve with given singularities. The parabola $y=9 \sqrt{x}$ goes through points corresponding to non-smooth $\mathrm{ESF}$, and the parabola $y=4 \sqrt{x}-4$ indicates a sufficient condition for the smoothness of ESF (4.3).

We conjecture that there cannot be an asymptotically optimal sufficient condition for the irreducibility of ESF.

One more conjecture concerns a possible form of sufficient conditions for the smoothness and irreducibility of ESF:

Conjecture 1 There exists a positive invariant $\beta$ of topological (analytic) singularities and an absolute constant $a>0$ such that

$$
\sum_{i=1}^{r} \beta\left(S_{i}\right) \leq a d^{2}
$$

is sufficient for the smoothness and the irreducibility of the corresponding topological (respectively analytic) ESF, and, in addition, there exists a sequence of curves of degrees $d \rightarrow \infty$ with the total $\beta$-invariant, growing faster than $d^{2}$. 


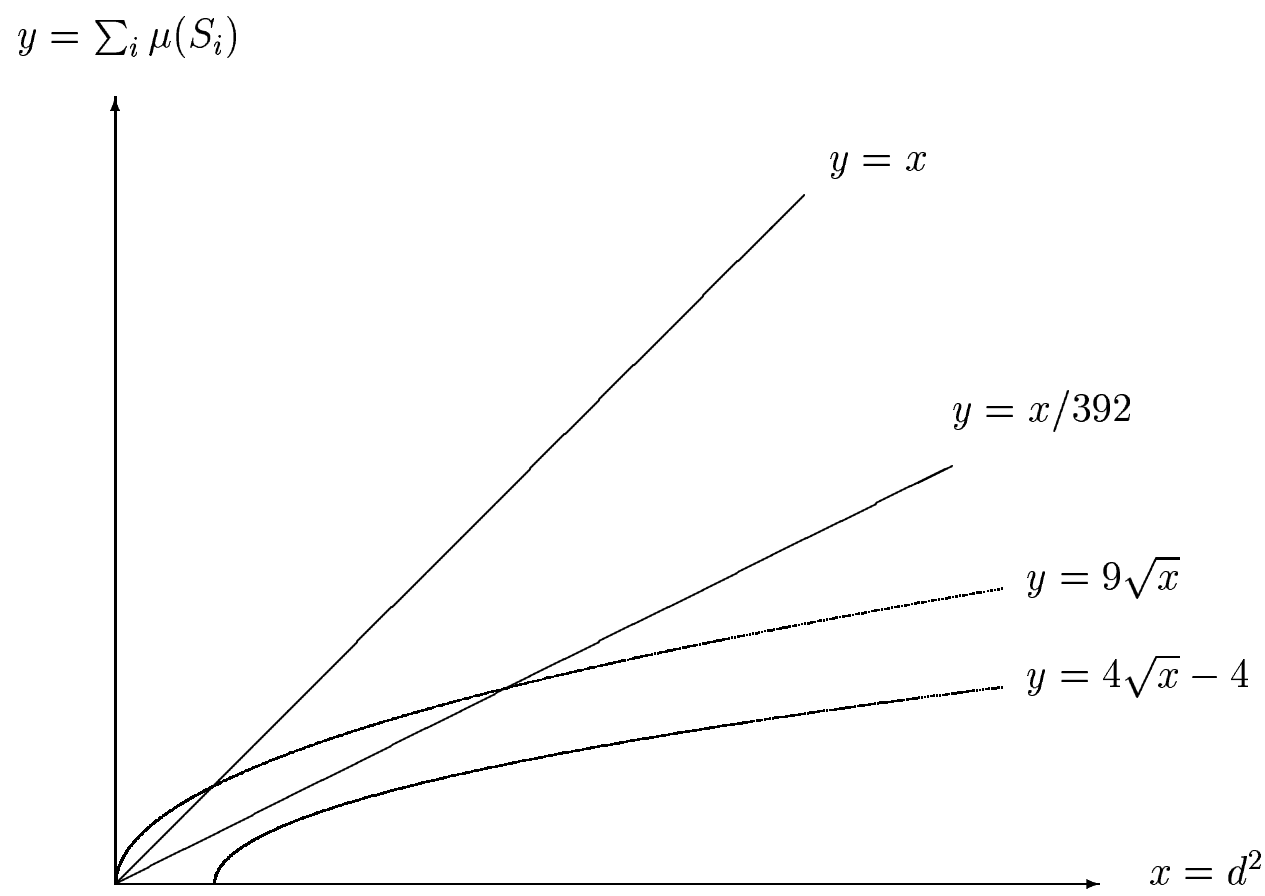

Figure 1: Necessary and sufficient conditions for existence and smoothness 
Condition (4.4) is weaker than any asymptotically optimal one, but is stronger that any condition of type (3.5) in the following sense: any condition of type (4.4) can be reduced to a condition of type (3.5), for instance,

$$
\sum_{i=1}^{r} \mu\left(S_{i}\right) \leq \min _{1 \leq i \leq r} \frac{\mu\left(S_{i}\right)}{\beta\left(S_{i}\right)} d^{2},
$$

but no condition of type (3.5) can be reduced to any condition of type (4.4).

(5) Given a smooth algebraic surface $\Sigma$ and a divisor $D$ on $\Sigma$, the problem about the geometry of ESF of curves in the linear system $|D|$ can be stated in the same way as for the plane: what are numerical necessary and sufficient conditions for the non-emptiness, smoothness and irreducibility of such ESF ? The known general sufficient condition for the smoothness of ESF

$$
\sum_{i=1}^{r}\left(\tau\left(S_{i}\right)-1\right)<-D K_{\Sigma}
$$

where $K_{\Sigma}$ is the canonical divisor, seems to be not optimal, since on the plane it turns into a "linear" condition (4.2). New results on families of nodal curves on $\mathbb{P}_{m}^{2}$ the projective plane blown up at $m>9$ generic points [29] and on nodal curves on surfaces of general type [14] cover a much wider range than (4.5). These new conditions can be formulated in an invariant form as follows. Assume that $\Sigma=\mathbb{P}_{m}^{2}$. Then the inequalities

$$
\begin{aligned}
n \leq \frac{A_{1}+B_{1}}{2}+ & 1-\frac{(m+9) B_{1}^{2}+9(m-9) A_{1}+6 \sqrt{m} B_{1} \sqrt{B_{1}^{2}+(m-9) A_{1}}}{2(m-9)^{2}} \\
& -\frac{3}{2(m-9)}\left(\sqrt{m} B_{1}+3 \sqrt{B_{1}^{2}+(m-9) A_{1}}\right)
\end{aligned}
$$

where $A_{1}=D^{2}, B_{1}=D K_{\Sigma}$,

$$
[\sqrt{2 n}]<\frac{(\sqrt{m}-3 \sqrt{2}) \sqrt{B_{2}^{2}+(m-9) A_{2}}-(\sqrt{2 m}-3) B_{2}}{2(m-9)},
$$

where $A_{2}=\left(D-2 K_{\Sigma}\right)^{2}, B_{2}=\left(D-2 K_{\Sigma}\right) K_{\Sigma}$,

$$
[\sqrt{2 n}] \leq \frac{\sqrt{B_{3}^{2}+(m-9) A_{3}}-B_{3}}{4(\sqrt{m}+3)}
$$

where $A_{3}=D^{2}, B_{3}=D K_{\Sigma}$, suffice that the family of curves with $n$ nodes in the linear system $|D|$ is non-empty, smooth and irreducible, respectively. Similarly, if $\Sigma$ is a surface of general type, then the Chiantini-Sernesi sufficient condition for the smoothness and the $T$-property of the family of curves with $n$ nodes in the linear system $|D|$ (see [14]) looks as

$$
n<\frac{D^{2}-2 D K_{\Sigma}+1}{4} .
$$

Comparing these results with the general necessary condition for the existence of an irreducible curve in $|D|$ on an arbitrary surface $\Sigma$ (see some stronger restrictions for special surfaces in [13], [91]):

$$
\sum_{i=1}^{r} \delta\left(S_{i}\right) \leq \frac{D^{2}+D K_{\Sigma}}{2}+1
$$


we conjecture that an asymptotically optimal form of a sufficient condition for the properties considered may be

$$
\sum_{i=1}^{r} \alpha\left(S_{i}\right) \leq \Phi\left(D^{2}, D K_{\Sigma}\right)
$$

with some positive invariant $\alpha$ of (topological or analytic) singularities and a function $\Phi$, linear in $D^{2}$ and quadratic in $D K_{\Sigma}$.

(6) Problems on the geometry of ESF of algebraic curves can be formulated in a similar way for equisingular families of hypersurfaces of a given degree in $\mathbb{P}^{m}, m \geq 3$, with given isolated singularities. In contrast to the existence problem, which conjecturally admits an approach coming from the study of curves discussed above, for the smoothness and irreducibility we do not have yet an adequate approach. The first step in this direction was made by Du Plessis and Wall [19]. They used the theory of discriminant matrices and obtained, in particular, the following result:

Lemma 5 If

$$
\frac{1}{m}+\frac{1}{d}>\frac{1}{2}
$$

and

$$
\sum_{i=1}^{r} \tau\left(S_{i}\right)<(d-1)^{m-1}(2 m+2 d-m d)
$$

then the family of hypersurfaces of degree $d$ in $\mathbb{P}^{m}$ with given analytic singularities $S_{1}, \ldots, S_{r}$ is smooth and has dimension

$$
\left(\begin{array}{c}
d+m \\
m
\end{array}\right)-1-\sum_{i=1}^{r} \tau\left(S_{i}\right)
$$

The inequality (4.6) restricts the application range to finitely many pairs $d, m \geq 3$. So the question how to handle the general case, with arbitrary $d, m$, remains still open and the most important in the problem discussed.

\section{References}

[1] Alexander J. and Hirschowitz A. Le methode d'Horace éclatée: application à l'interpolation en degrée quatre. Invent. Math. 107 (1992), 585-602.

[2] Arbarello E. and Cornalba M. A few remarks about the variety of irreducible plane curves of given degree and genus. Ann. sci. Ec. norm. super. 16 (1983), no. 3, 467-488.

[3] Arnol'd V.I., Gusein-Zade S.M., and Varchenko A.N. Singularities of differentiable maps, vol.1. Boston, Basel, Stuttgart: Birkhäuser Verlag, 1985.

[4] Artin M. Versal deformations and algebraic stalks. Invent. Math. 27 (1974), 165-189.

[5] Artin M. Deformations of singularities. Tata Institute of Fundamental Research, Bombay, 1976.

[6] Barkats D. Non vacuité des variétés des courbes planes a noeuds et a cusps. Preprint no. 363, Université de Nice-Sophia-Antipolis, December 1993. 
[7] Barkats D. Irreducibilité des variétés des courbes planes a noeuds et a cusps. Preprint no. 364, Université de Nice-Sophia-Antipolis, December 1993.

[8] Barkats D. Private communication.

[9] Brieskorn E. and Knörrer H. Plane algebraic curves. Birkhäuser-Verlag, 1986.

[10] Bruce J. W. Singularities on a projective hypersurface. Bull. Lond. Math. Soc. 13 (1981), 47-50.

[11] Bruce J. W. and Gibblin P. J. A stratification of the space of plane quartic curves. Proc. London Math. Soc. 42 (1981), 270-298.

[12] Brusotti L. Sulla "piccola variazione" di una curva piana algebrica reali. Rend. Rom. Ac. Lincei (5) 30 (1921), 375-379.

[13] Chang M. C. and Ran Z. Divisors on some generic hypersurfaces. J. Diff. Geom. 38 (1993), 671-678.

[14] Chiantini L. and Sernesi E. Nodal curves on surfaces of general type. Math. Ann. 307 (1997), 41-56.

[15] Davis E. D. 0-dimensional subschemes of $\mathbb{P}^{2}$ : new applications of Castelnuovo's function. Ann. Univ. Ferrara 32 (1986), 93-107.

[16] Degtyarev A. Isotopy classification of complex plane projective curves of degree five. Preprint P-3-87, Steklov Math. Inst., Leningrad branch, 1987 (Russian).

[17] Degtyarev A. Quintics in $\mathbb{C}^{2}$ with non-abelian fundamental group. Preprint, MaxPlanck-Institut für Mathematik, 1995.

[18] Diaz S., and Harris J. Ideals associated to deformations of singular plane curves. Trans. Amer. Math. Soc. 309 (1988), no. 2, 433-468.

[19] Du Plessis A. A. and Wall C. T. C. Versal deformations in spaces of polynomials of fixed weight. Preprint no. 16, University of Aarhus, July 1996.

[20] Du Plessis A. A. and Wall C. T. C. The Geometry of Topological Stability. Clarendon Press, Oxford, 1995.

[21] Finashin S. and Shustin E. On imaginary plane curves and Spin quotients of complex surfaces by complex conjugation. Amer. Math. Soc. Transl., to appear.

[22] Giacinti-Diebolt C. Variétés des courbes projectives planes de degrée et lieu singulier donnés. Math. Ann. 266 (1984), no. 3, 321-350.

[23] Gradolato M. and Mezzetti E. Curves with nodes, cusps and ordinary triple points. Ann. Univ. Ferrara, sez. 7, 31 (1985), 23-47.

[24] Gradolato M.A. and Mezzetti E. Families of curves with ordinary singular points on regular surfaces. Ann. mat. pura ed appl. 150 (1988), 281-298. 
[25] Greuel, G.-M. A remark on the paper of A. Tannenbaum. Composito Math. 51, 185-187 (1984).

[26] Greuel G.-M. and Karras U. Families of varieties with prescribed singularities. Compos. math. 69 (1989), no. 1, 83-110.

[27] Greuel G.-M. and Lossen C. Equianalytic and equisingular families of curves on surfaces. Manuscr. Math. 91 (1996), 323-342.

[28] Greuel G.-M., Lossen C. and Shustin E. Plane curves of minimal degree with prescribed singularities. Submitted to Invent. Math.

[29] Greuel G.-M., Lossen C. and Shustin E. Families of nodal curves on the blown-up projective plane. Submitted to Trans. Amer. Math. Soc.

[30] Greuel, G.-M., Pfister, G., Hertling, C. Moduli spaces of semiquasihomogeneous singularities with fixed principal part. To be published in J. Algebraic Geometry.

[31] Greuel G.-M., and Pfister G. Moduli for singularities. In: Singularities/London Math. Soc. Lect. Notes 201, J.-P. Brasselet, ed., Cambridge Univ. Press, Cambridge, 1994, pp. 119-146.

[32] Greuel, G.-M., and Pfister, G. Geometric quotients of unipotent group actions. Proc. London Math. Soc. (3) 67, 75-105 (1993).

[33] Greuel, G.-M. and Pfister G. On moduli spaces of semiquasihomogeneous singularities. Progress in mathematics 134, 171-185, Birkhäuser 1996.

[34] Greuel, G.-M. and Pfister, G. Geometric quotients of unipotent group actions II. Preprint, Univ. of Kaiserslautern 1995. To be published in "Singularities", Festband on the occasion of Brieskorn's 60th birthday.

[35] Greuel, G.-M., Pfister, G. and Schönemann, H. SINGULAR, A Computeralgebra system for Algebraic Geometry and Singularity theory. Available via ftp from helios.mathematik.uni-kl.de.

[36] Gudkov D. A., Tai M. L., and Utkin G. A. Complete classification of irreducible curves of the 4th order. Mat. Sbornik 69 (1966), no. 2, 222-256 (Russian).

[37] Gudkov D.A. Topology of real projective algebraic varieties. Rus. Math. Surveys 29 (1974), no. 4, 3-79.

[38] Harbourne B. Complete linear systems on rational surfaces. Trans. Amer. Math. Soc. 289 (1985), 213-226.

[39] Harris J. On the Severi problem. Invent. Math. 84 (1985), 445-461.

[40] Hirano A. Constructions of plane curves with cusps. Saitama Math. J. 10 (1992), 21-24.

[41] Hirschowitz A. Une conjecture pour la cohomologie des diviseurs sur les surfaces rationelles génériques. $J$. reine angew. Math. 397(1989), 208-213. 
[42] Hirzebruch F. Singularities of algebraic surfaces and characteristic numbers. Contemp. Math. 58 (1986), 141-155.

[43] Itenberg I. and Shustin E. Real algebraic curves with real cusps. Amer. Math. Soc. Transl. (2) 173 (1996), 97-109.

[44] Ivinskis K. Normale Flächen und die Miyaoka-Kobayashi Ungleichung. Diplomarbeit, Bonn, 1985.

[45] Kang P.-L. On the variety of plane curves of degree $d$ with $\delta$ nodes and $k$ cusps. Trans. Amer. Math. Soc. 316 (1989), no. 1, 165-192.

[46] Kang P.-L. A note on the variety of plane curves with nodes and cusps. Proc. Amer. Math. Soc. 106 (1989), no. 2, 309-312.

[47] Korchagin A.B. and Shustin E. Affine curves of degree 6 and smoothings of the ordinary 6th order singular point. Math. USSR Izvestia 33, N.3, 501-520 (1989).

[48] Laudal A. Formal moduli of algebraic structures. (Lecture Notes Math., vol. 754), Berlin etc, Springer, 1979.

[49] Laudal O.A., Martin B., and Pfister G. Moduli of plane curve singularities with $\mathbb{C}^{*}$ action. In: Banach Cent. Publ. Vol. 20. Singularities. Warszawa, 1988, pp. 255-278.

[50] Lindner M. Über Mannigfaltigkeiten ebener Kurven mit Singularitäten. Arch. Math. 28 (1977), 603-610.

[51] Loeser I. Deformations des courbes planes. Seminaire Bourbaki, 1986-87, no. 679.

[52] Luengo I. The $\mu$-constant stratum is not smooth. Invent. Math. 90 (1987), 139-152.

[53] Nobile A. Families of curves on surfaces. Math. ZeitschriftZ 187 (1984), no. 4, 453-470.

[54] Pecker D. On the geometric genus of projective curves. Preprint, Université Pierre \& Marie Curie, 1996.

[55] Ran Z. Families of plane curves and their limits: Enriques' conjecture and beyond. Annals of Math. 130 (1989), no. 1, 121-157.

[56] Sakai F. Singularities of Plane Curves. In: Geometry of Complex Projective Varieties, Seminars and Conferences 9, Mediterranian Press, Rende, 1993, pp. 257-273.

[57] Santos F. Construction of real algebraic plane curves. Proc. Real Algebraic and Analytic Geometry, Trento, Sept. 1992, Berlin, Walther de Gruyter, 1993, pp. 213-228.

[58] Schlessinger M. Functors of Artin rings. Trans. AMS 130 (1968), 208-222.

[59] Segre B. Dei sistemi lineari tangenti ad un qualunque sistema di forme. Atti Acad. naz. Lincei Rendiconti serie 5, 33 (1924), 182-185.

[60] Segre B. Esistenza e dimensione di sistemi continui di curve piane algebriche con dati caraterri. Atti Acad. naz. Lincei Rendiconti serie 6, 10 (1929), 31-38. 
[61] Severi, F. Vorlesungen über Algebraische Geometrie (Anhang F). Leipzig, Teubner, 1921.

[62] Shustin E. New M-curve of the 8th degree. Math. Notes of Acad. Sci. USSR 42 (1987), 606-610.

[63] Shustin E. Versal deformations in the space of plane curves of fixed degree. Function. Anal. Appl. 21 (1987), 82-84.

[64] Shustin E. Geometry of discriminant and topology of algebraic curves. Proc. Internat. Congr. of Math., Kyoto, Japan, Aug. 21-29, 1990, vol. 1, Springer, 1991, pp. 559-567.

[65] Shustin E. Hyperbolic and minimal smoothings of singular points. Selecta Math. Sov. 10 (1991), no.1, 19-25.

[66] Shustin E. On manifolds of singular algebraic curves. Selecta Math. Sov. 10 (1991), no.1, 27-37.

[67] Shustin E. Real plane algebraic curves with prescribed singularities. Topology 32 (1993), no. $4,845-856$.

[68] Shustin E. Smoothness and irreducibility of varieties of algebraic curves with nodes and cusps. Bull. SMF 122 (1994), 235-253.

[69] Shustin E. Smoothness and irreducibility of families of plane algebraic curves with ordinary singularities. In: Israel Mathematical Conference Proceedings, vol. 9, AMS, Providence Rh. I., 1996, pp. 393-416.

[70] Shustin E. Geometry of equisingular families of plane algebraic curves. J. Algebraic Geometry 5 (1996), no. 2, 209-234.

[71] Shustin E. Smoothness of equisingular families of plane algebraic curves. International Math. Research Notices, to appear.

[72] Shustin E. Gluing of singular and critical points. Topology, to appear.

[73] Shustin E. and Tyomkin I. Linear systems of plane curves with generic multiple points. Preprint 4-96, Tel Aviv University, May 1996.

[74] Tannenbaum A. Families of algebraic curves with nodes. Composito Math. 41 (1980), 107-126.

[75] Tannenbaum A. Families of curves with nodes on K3-surfaces. Math. Ann. 260 (1982), 239-253.

[76] Tannenbaum A. On the classical characteristic linear series of plane curves with nodes and cuspidal points: two examples of Beniamino Segre. Composito Math. 51 (1984), 169-183.

[77] Teissier B. The hunting of invariants in the geometry of discriminants. In: Real and Complex Singularities./ P.Holm, editor. Oslo: Sijthoff-Noordhoff Publ., Alphen aan den Rijn, 1977, p. 565-677. 
[78] Tougeron J. C. Ideaux des fonctions différentiables. Ann. Inst. Fourier (Grenoble) 18 (1968), no. 1, 177-240.

[79] Urabe T. On quartic surfaces and sextic curves with certain singularities. Proc. Japan. Acad. Ser. A. 59 (1983), 434-437.

[80] Urabe T. On quartic surfaces and sextic curves with singularities of type $\widetilde{E}_{8}, T_{2,3,7}, \widetilde{E}_{12}$. Publ. Inst. Math. Sci. Kyoto Univ. 80 (1984), 1185-1245.

[81] Urabe T. Singularities in a certain class of quartic surfaces and sextic curves and Dynkin graphs. Proc. Vancouver Conf. Algebraic Geom., July 2-12, 1984. Providence, 1986, p. 477-497.

[82] Varchenko A.N. Asymptotic of integrals and Hodge structures. In: Modern Problems of Math., Vol. 22 (Itogi nauki i tekhniki VINITI), Moscow, 1983, pp. 130-166 (Russian).

[83] Vassiliev V.A. Stable cohomology of complements to the discriminant of deformations of singularities of smooth functions. J. Soviet Math. 52 (1990), no. 4, 3217-3230.

[84] Viro O. Ya. Gluing of algebraic hypersurfaces, smoothing of singularities and construction of curves. Proc. Leningrad Int. Topological Conf., August 1983, Nauka, Leningrad, 1983, pp. 149-197 (Russian).

[85] Viro O. Ya. Gluing of plane real algebraic curves and construction of curves of degrees 6 and 7. Lect. Notes Math. 1060, Springer, Berlin etc., 1984, pp. 187-200.

[86] Viro O.Ya. Real algebraic plane curves: constructions with controlled topology. Leningrad Math. J. 1 (1990), 1059-1134.

[87] Wahl J. Deformations of plane curves with nodes and cusps. Amer. J. Math. 96 (1974), 529-577.

[88] Wahl J. Equisingular deformations of plane algebroid curves. Trans. Amer. Math. Soc. 193 (1974), 143-170.

[89] Wall C. T. C. Geometry of quartic curves. Math. Proc. Camb. Phil. Soc. 117 (1995), 415-423.

[90] Wall C. T. C. Highly singular quintic curves. Math. Proc. Camb. Phil. Soc. 119 (1996), 257-277.

[91] Xu G. Subvarieties of general hypersurfaces in projective spaces. J. Diff. Geom. 39 (1994), 139-172.

[92] Zariski O. Studies in equisingularity I-III. Amer. J. Math. 87 (1965), 507-536, Amer. J. Math. 87 (1965), 972-1006, and Amer. J. Math. 90 (1968), 961-1023.

[93] Zariski O. Algebraic surfaces. 2nd ed. Heidelberg, Springer, 1971. 